\title{
REVIEWS
} Monika Pazgan-Simon ${ }^{4,}$ B-C, Brygida Knysz ${ }^{1, \text { A, E-F }}$

\section{Current Possibilities to Assess the Degree of Liver Fibrosis in Patients with Haemophilia Infected with HCV - Review}

\footnotetext{
${ }^{1}$ Department of Infectious Diseases, Hepatology and Acquired Immune Deficiences, Wrocław Medical University, Poland

${ }^{2}$ Department of General and Pediatric Radiology, Wroclaw Medical University, Poland

${ }^{3}$ Department of Hematology, Blood Neoplasms and Bone Marrow Transplantation, Wrocław Medical University, Poland

${ }^{4} 1^{\text {st }}$ Division of Infectious Diseases, J. Gromkowski Regional Specialist Hospital, Wrocław, Poland
}

A - research concept and design; $\mathbf{B}$ - collection and/or assembly of data; C - data analysis and interpretation;

$\mathbf{D}$ - writing the article; $\mathbf{E}$ - critical revision of the article; $\mathbf{F}$ - final approval of article; $\mathbf{G}$ - other

\begin{abstract}
Haemophilia is an entity, wherein the HCV infection rate is greater than in the general population and ranges between $70-90 \%$. The majority of HCV infections were acquired by hemophiliacs in the 1980s, by the use of infected cryoprecipitate or fresh frozen plasma preparations. It is therefore highly likely that many of them, more than twenty years after the infection, have developed advanced liver disease. Until recently, in order to assess its severity, it was necessary to perform a liver biopsy. Currently, we observe rapid developments of non-invasive methods that are particularly useful in patients with bleeding disorders. The most popular include elastography (Fibroscan, SWE) and the algorithms based on measurements of the relevant blood parameters (e.g. FibroTest, Fibrometer, APRI index). Ease of implementation of these studies, no need for hospitalization of the patient or specific preparation for surgery, allow for quick and minimally invasive assessment of liver disease progression and classification of the patient to the appropriate antiviral therapy (Adv Clin Exp Med 2015, 24, 4, 671-677).
\end{abstract}

Key words: fibrosis, haemophilia, hepatitis C, non-invasive methods, liver biopsy.

\section{Haemophilia and HCV Infection}

Haemophilia is a disease in which the incidence of HCV infection is higher than in the general population and ranges from $70-90 \%[1,2]$. The incidence in the Polish population is comparable to that observed in the United States and Western Europe. The study of Windyga et al. which was carried out on 172 patients born between 1935-1990 (mean age $35.3 \pm 11.6$ ), diagnosed with severe haemophilia A or $\mathrm{B}$, who had received intravenous infusions of fresh frozen plasma, cryoprecipitate and blood before 1991, demonstrated that $95 \%$ of respondents had been infected with hepatitis C. More than 77\% of them have not eliminated the infection and continue to actively replicate the virus. It is therefore a significantly higher incidence as compared to the general population and analogous to the above-cited statistic [3]. The percentage of HCV-infected persons in Poland is estimated at $1.5 \%$. It can be assumed that in our country there are approximately 1,000 patients with severe haemophilia and chronic hepatitis $\mathrm{C}$ infection.

The majority of HCV infections was acquired by hemophiliacs in the 1980s, by the use of infected cryoprecipitate or fresh frozen plasma preparations, which did not undergo any procedures for the inactivation of infectious particles at that time. Chronic hepatitis $\mathrm{C}$ infection leads to progressive liver fibrosis, cirrhosis and hepatocellular carcinoma [4]. It is therefore highly likely that many patients, more than twenty years after the infection, have developed advanced liver disease. 


\section{Methods of Evaluation of Liver Fibrosis}

Previously, in order to assess the severity of liver disease, it was necessary to perform liver biopsy procedure. For several years, we have seen rapid developments of non-invasive methods of evaluating fibrosis and inflammation in the liver. They are particularly useful in patients with contraindications to performing invasive procedures, including patients with bleeding disorders.

\section{Liver Biopsy}

Liver biopsy remains the "gold standard" in assessing the severity of liver damage. Histopathological examination, obtained by biopsy, assesses the degree of fibrosis and the severity of inflammation in the liver. It is also an indispensable diagnostic tool that allows to further diagnose the ongoing intrahepatic processes, including autoimmune reactions, copper or iron-storage [5]. However, it is an invasive procedure. The complication rate is estimated at $0.3-0.6 \%$, and the mortality rate at $0.05 \%$ [6]. The most common complications of the procedure are pain and asymptomatic bleeding (subcapsular and intracapsular hematomas). Very rarely, there is bleeding into bile ducts, puncture of other organs, like lungs (to form a pneumothorax, subcutaneous edema, pleural effusion) kidney, colon or gallbladder [7].

A significant limitation of liver biopsy is the chance of puncturing of non-diagnostic sample. Its representative length should be $1.0 \mathrm{~cm}$. Obtaining a shorter sample limits the correct interpretation, especially the reliable assessment of the degree of fibrosis. Obtaining a reliable biopsy sample may be difficult also because of the varying degree of fibrosis in the liver and depending on the location of the sample. Regev et al. demonstrated that non-uniformly extending liver fibrosis is a potential limitation of the liver biopsy. In this study on a group of 124 patients with chronic hepatitis $\mathrm{C}$ infection liver biopsies obtained laparoscopically from both the right and the left lobe of the liver were histologically assessed. Significant differences were shown in 41 patients (33.1\%) in the results of the assessment of fibrosis (at least one degree of difference in terms of fibrosis according to the Scheuer's scale) [8]. Bedossa et al., in turn, demonstrated that a biopsy sample length of a minimum of $25 \mathrm{~mm}$ increases the accuracy of the assessment of fibrosis by partial eliminating the above-mentioned limitations [9]. They also showed that in up to one third of respondents histological sections may be misdiagnosed $[8,9]$. Therefore, the liver biopsy, because of the above-mentioned limitations, primarily its invasiveness, is a mediocre tool in assessing the dynamics of the degree of organ damage.

\section{Non-Invasive Methods}

Consequently, there is a growing interest in using non-invasive methods to assess the degree of fibrosis and inflammation in the liver. Easy application and no need for hospitalization of the patient or specific preparation for surgery allow for a quick and minimally invasive assessment of liver disease progression, and classifying the patient to the appropriate antiviral therapy. In addition, several studies have shown the advantages of these methods over liver biopsy in the assessment of the degree of liver damage $[10,11]$. In the study of Poynard et al., the degree of compatibility between non-invasive methods such as Fibrotest and ActiTest and liver biopsy was evaluated [11]. A discrepancy was revealed in the results in $29 \%$ of patients, with $18 \%$ resulting from erroneous biopsy of the liver, and only $2.4 \%$ from the interpretation of non-invasive testing methods. Nevertheless, histopathological examination of the liver remains up to date the decisive method.

\section{Elastography}

\section{Fibroscan}

Fibroscan (TE - transient elastography; onedimensional transient elastography) is an increasingly commonly used elastographic method, serving non-invasive measurement of the degree of stiffness of the liver, indirectly assessing the degree of fibrosis. It involves assessing the velocity of propagation of an elastic shear wave generated by the mechanical impulse from applied transducer. This rate depends on the cohesiveness of the liver, and the degree of cohesiveness increases in direct proportion to the degree of fibrosis. The velocity measurements are computer-processed and the final result is the average of 10 measurements. Known limitations of this method are: a significant degree of obesity, pregnancy, and the presence of ascites [12].

It has been shown that there is a good correspondence between this method and the stage of liver fibrosis $[13,14]$. In the most recent recommendations of the European Association for the Study of the Liver elastography is mentioned as a method with a high degree of reliability in assessing the severity of chronic hepatitis C [15]. In Poland since 2011, according to the regulation of the Ministry of Health, this study can be used as an alternative to liver biopsy in the assessment of 
eligibility of patients for treatment programs of chronic hepatitis B and C. Many authors believe that this is the most appropriate tool for diagnosing liver cirrhosis. In earlier stages of liver fibrosis the differences between obtained results are less pronounced $[16,17]$.

\section{Shear-Wave Elastography}

Shear-wave elastography (SWE) is the latest method of assessing the severity of fibrosis. Like one-dimensional transient elastography (TE), it is based on the measurement of the velocity of the induced shear wave in the imaged tissues. The essence of SWE is to generate a number of shear waves at different depths, which interfere with each other to form a cone-like shape shear wave. Due to a very high speed of acquisition the velocity of propagation of the interference wave can be measured. The software then generates a map of the velocity of propagation of the wave and calculates the relative hardness of the organ. The measurement results are expressed in $\mathrm{kPa}$ or $\mathrm{m} / \mathrm{s}$ and also visualized by applying a color image on a conventional ultrasound image. The operator performs several measurements, with mean value calculated, on the basis of which (as in the case of Fibroscan) the degree of fibrosis is determined using Metavir scale. The advantages of this method are much independence from the investigator, its speed and overall safety of the patient. In addition, the operator is able to assess the morphology of the organ (to detect potential focal changes) and, if necessary, measurements of flow in the portal vein system can be performed. The operator can also choose the best places, where the measurements will be made (avoiding large vessels or capsula of the liver), which further increases the reliability of the study. Use of the ultrasound beam to produce a transverse wave and ability to choose the location of the measurement the stiffness of the tissue allows us also to examine patients with ascites. As in the case of the transient elastography (Fibroscan), shear wave elastography is difficult to perform in patients with significant obesity. Several studies have confirmed the high diagnostic efficacy of the SWE method and compliance of the results of the biopsy, TE and FibroTest $[18,19]$.

\section{Magnetic Resonance Elastography}

Magnetic resonance elastography is another radiological method allowing for the quantitative measurement of liver stiffness and the assessment of the degree of fibrosis. The velocity of propagation of the mechanical stimulus (wave) in the examined tissue is measured with the help of magnetic resonance. Based on the collected data, the software calculates the hardness of the liver and allows us to determine the degree of fibrosis. MRE option is available as an add-on software to conventional MRI scanners. An important advantage of this method is complete independence from the operator, and the ability to make measurements in the whole organ, and not a few selected points. The disadvantages include the long duration of the study, the high price of software and difficulties in availability of this method $[20,21]$.

\section{FibroTest}

FibroTest is another method, an alternative to the liver biopsy, which can be used in patients with chronic hepatitis $\mathrm{C}$. The method involves determining serum alfa 2-macroglobulin, haptoglobin, apoliprotein A-1, total bilirubin, GGTP, and then calculating (patent protected) the indicator of fibrosis. In addition, the determination of GTP allows us to perform an ActiTest, evaluating necroinflammatory activity in chronic viral hepatitis. The limitations of this method concern disorders in which there is a decrease or increase of the value of these parameters. This might include: severe hemolysis, acute hepatitis, massive hepatic necrosis, acute inflammation associated with viral or bacterial infection, extrahepatic cholestasis and hypercholesterolemia with high concentrations of HDL. The value of the method is determined by the value of AUROC (area under the receiver operating characteristic), which also allows us to assess its sensitivity and specificity. In the case of FibroTest AUROC $=0.84$ is achieved as compared to the results obtained by liver biopsy [22, 23].

\section{Other Non-Invasive Methods}

Other non-invasive methods applied in chronic hepatitis $\mathrm{C}$ include determination of serum fibrosis markers or more complex algorithms taking into account the values of the selected markers. The former are divided into two groups: direct and indirect ones. Direct markers of fibrosis include: (1) the concentration of hyaluronic acid [24], (2) laminin [25], (3) some of the collagen proteins (such as PNP III, type IV collagen), [26] (4) collagenase or their inhibitors (e.g. MMP, TIMP-1) $[27,28]$. The latter include, among others, (1) the number of platelets (2) prothrombin activity, (3) the GOT/GPT ratio.

There are also algorithms based on the calculation of the relevant parameters of blood. These include: (1) APRI index, taking into account the activity of GOT and platelet count, (2) FIB-4, which also takes into account the age of the patient. There are also more complex algorithms, for instance Fibrometer, which takes into account 
the values of parameters such as platelet count, prothrombin time, GOT, alpha 2-macroglobulin concentration, hyaluronic acid, urea, and age. Another algorithm of this type is the Hepascore model. It is based on the calculation of parameters such as serum bilirubin, GGTP, alpha 2-macroglobulin, hyaluronic acid, the age and sex of the

Table 1. Selected non-invasive models to assess liver fibrosis

\begin{tabular}{|l|l|}
\hline FibroTest & $\begin{array}{l}\text { alfa 2-macroglobulin, GGTP, } \\
\text { apolipoprotein A-1, haptoglobin, } \\
\text { total bilirubin, age, sex }\end{array}$ \\
\hline APRI & GOT, PLT \\
\hline Hepascore & $\begin{array}{l}\text { bilirubin, GGTP, hyaluronic } \\
\text { acid, sex, age }\end{array}$ \\
\hline Fibrometer & $\begin{array}{l}\text { PLT, prothrombin time, GOT, } \\
\text { alfa 2-macroglobulin, hyaluronic } \\
\text { acid, urea, age }\end{array}$ \\
\hline Forns Index & age, PLT, cholesterol, GGTP \\
\hline ELF (Enhanced & $\begin{array}{l}\text { age, hyaluronic acid, MMP-3, } \\
\text { TiMP-1 }\end{array}$ \\
\hline FibroSpect & $\begin{array}{l}\text { alfa 2-macroglobulin, hyaluronic } \\
\text { acid, TIMP-1 }\end{array}$ \\
\hline MP3 & MMP-3, TIMP-1 \\
\hline FIB-4 & ALT, AST, PLT, age \\
\hline
\end{tabular}

patient $[29,30]$. Table 1 shows the most popular models.

AUROC values for the above methods in advanced liver fibrosis are 0.72-0.78, and are slightly higher in cirrhosis, AUROC between $0.77-0.86$.

Limitations of these methods stem from a lack of specific markers for liver fibrosis. Either an increase or decrease of the mentioned parameters for reasons independent of liver fibrosis, can significantly affect the test result. An example is the increased bilirubin level in patients with Gilbert's syndrome, lower concentration of haptoglobin in patients with hemolysis, or increased concentration of hyaluronic acid after a meal or in patients with chronic inflammatory processes such as rheumatoid arthritis. Studies have shown that hypercholesterolemia can lead to a false result of Forns index. It is based on the calculated parameters such as platelet count, age, GGTP and cholesterol level. In contrast, normal GOT activity may prevent the proper assessment of fibrosis by APRI index. Hence, it is difficult to assess the overall consistency of results of liver fibrosis, which is obtained by different methods.

\section{A Comparative Study of Methods to Assess Liver Fibrosis}

The study of Caster et al. found the compatibility of the results obtained by two methods, i.e. using FibroTest and TE (Fibroscan), ranging from 70 to $79 \%$ [31]. It was also shown that if the results of both methods are consistent, then a liver biopsy confirms the previously established diagnosis in $84 \%$ of cases for $\mathrm{F} \geq 2$, in $95 \%$ for $\mathrm{F} \geq 3$, and in $94 \%$ for $\mathrm{F}=4$, respectively (based on a 5-point METAVIR scale, where $\mathrm{F}=0$ indicates no fibrosis, $\mathrm{F}=4$ is liver cirrhosis). It was found thus that the combination of both non-invasive methods assesses hepatic fibrosis more accurately than in the case when each of them is used separately. [37].

Similar results were obtained in a study by Mayor et al., in which histopathological examination of the liver was not performed, and non-invasive methods i.e. TE and Fibrotest were used to assess the degree of liver damage. The study demonstrated a gradual increase in the rate of compliance index in ever more advanced stages of fibrosis. When fibrosis was assessed for $\mathrm{F} \geq 2$, for $\mathrm{F} \geq 3$ and for $\mathrm{F}=4$, this index was $62 \%, 69 \%$ and $85 \%$, respectively [32].

The study of Ferraioli et al. rated the accuracy of results obtained by both elastographic methods i.e. FibroScan (TE) and SWE elastography, compared to results obtained by liver biopsy. This was the first study comparing the two elastographic methods with liver biopsy performed on the same day. The study was performed on a group of 121 patients with chronic hepatitis $\mathrm{C}$ infection. $\mathrm{Pa}$ tients co-infected with HIV, with overt cirrhosis and with ascites were excluded from the study. It has been shown that SWE is more accurate in assessing of significant fibrosis i.e. for $\mathrm{F} \geq 2$ as compared to TE study, although there was no significant difference in the accuracy of assessment of tissue stiffness for advanced fibrosis for $\mathrm{F} \geq 3$. Moreover, the SWE correctly assessed the severity of fibrosis in $83.1 \%$ of patients, and TE in $66.7 \%$ of patients, while the discrepancy in results between TE and liver biopsy was probably a result of an incorrect result of histological examination [33].

Poynard et al. compared the results obtained by both elastographic methods, i.e. TE and SWE, and FibroTest. The study was conducted in 422 patients. It was shown that the most appropriate method was FibroTest, and the value of the 2 elastographic methods was comparable ("applicability" $-97.9 \%$ for the FT vs about $90 \%$ for the TE and SWE). In the case of liver cirrhosis results obtained 
by all methods were comparable, while in patients with ascites, SWE method was more useful than TE [34]. Further, it has been demonstrated that it is useful to combine different non-invasive methods in order to increase the accuracy of the result. This gives the opportunity to withdraw from performing a liver biopsy in situations where there is a risk of significant complications.

In the observations made by Boulier et al. of a group of 235 patients with chronic hepatitis C, hepatic fibrosis was evaluated by noninvasive tests such as FT, APRI, Forns index and by histological examination of liver biopsy sample. All tests were performed on the same day. It was shown that the overall consistency of the results using all 4 methods was obtained only in $29 \%$ of patients, while using only non-invasive methods - in 35\% of patients. The use of all three methods on the other hand made it possible to establish correct non-invasive assessment of fibrosis in 191 patients (81.3\%), reducing the need for a biopsy to 44 patients (18.7\%).

Similar observations were obtained in the SAFE (Sequential Algorithm for Fibrosis Evaluation) study. APRI index and FibroTest were used to determine the degree of liver fibrosis. An increase in precision of the test of up to $90 \%$ was achieved, thus reducing the need for liver biopsy by $47 \%$ of the patients [35].

Another way to strengthen the reliability of the measurement of liver fibrosis by non-invasive methods is the use of practical algorithms. In the available publications, there are primarily 2 of them discussed - Halfon algorithm and IQR/LSM ratio [11]. The former has been shown to have a high predictive value as to the accuracy of the determinations by FibroTest. According to this algorithm, if the result of the assessment of fibrosis obtained by FibroTest is in conformity with the result of APRI or Forns index result, it can be relied on. The latter algorithm, proposed by Lucidarme et al, was developed after analyzing factors responsible for the inconsistency of the results obtained from liver biopsy and elastography [36]. It is an indicator allowing for improved reliability of results obtained by FibroScan. Using these 2 different algorithms, Mayor et al. have created another one, which they claim allows reliable assessment of the anatomic condition of the organ in almost $87 \%$ of subjects with severe fibrosis [38].

\section{Assessment of Liver Fibrosis in Patients with Haemophilia Infected with $\mathrm{HCV}$}

Although liver biopsy is not absolutely contraindicated in patients with hemophilia, and studies did not show an increased number of complications in this group of patients, this procedure is much less frequently performed in patients with bleeding disorders [37]. It involves additional costs of hospitalization, resulting from the need of transfusion of the respective coagulation factors. In view of the fact that non-invasive methods are being used more and more often, individuals with bleeding disorders have a better chance of diagnosis, without having to undergo a liver biopsy.

In the study of Mayor et al., FibroTest was used to evaluate hepatic fibrosis in 132 patients with haemophilia and positive anti-HCV antibodies. It has been shown that in patients infected with HCV with underlying bleeding disorder advanced fibrosis at the level of F3 was present in $13 \%$ of patients, and liver cirrhosis in 17\% [38]. Similar results were obtained by Posthouwer et al., who conducted a study in 124 patients with chronic hepatitis C (average duration of infection was 34 years, range $14-40$ years). They used an elastographic method, finding cirrhosis in $17 \%$ of patients, and advanced fibrosis in $18 \%$ of patients [39]. This was much more than suggested by the results of laboratory tests and ultrasound. Based on these latter studies, the presence of liver cirrhosis was estimated in only $7 \%$ of respondents.

\section{Summary}

Opportunities of assessing liver fibrosis by non-invasive methods are growing. Therefore, it seems sensible to use them primarily in patients with bleeding disorders. While currently there are no strict guidelines as to the choice of method in haemophiliac patients, the validity of combining different non-invasive methods and the use of practical algorithms is being emphasized in order to overcome their shortcomings. None of the studies used individually can be perceived as the gold standard.

\section{References}

[1] Yee TT, Griffioen A, Sabin CA, Dusheiko G, Lee CA: The natural history of HCV in a cohort of haemophilic patients infected between 1961 and 1985. Gut 2000, 47, 845-851.

[2] Lee C, Dusheiko G: The natural history and antiviral treatment of hepatitis C in haemophilia. Heamophilia 2002, 8,322 . 
[3] Windyga J, Grabarczyk P, Stefańska E, Buczma A, Szczepanik AB, Klukowska A, Mikulska M, Medyńska J, Brojer E: Prevalence of HCV, HBV and HIV infections among severe Polish haemophiliacs. Przegl Epidemiol 2008, 62, 415-423.

[4] Fassio E: Hepatitis C and hepatocellular carcinoma. Ann Hepatol 2010, 9, 119-122.

[5] Dienstag JL: The role of liver biopsy in chronic hepatitis C. Hepatology 2002, 36, 152-160.

[6] Cadranel JF, Rufat P, Degos F: Practices of liver biopsy in France: results of a prospective nationwide survey. For the Group of Epidemiology of the French Association for the Study of the Liver (AFEF). Hepatology 2000, 32, 477-481.

[7] Grant A, Neuberger J: Guidelines on the use of liver biopsy in clinical practice. Gut 1999, 45, 1-15.

[8] Regev A, Berho M, Jeffers LJ, Milikowski C, Molina EG, Pyrsopoulos NT, Feng ZZ, Reddy KR, Schiff ER: Sampling error and intraobserver variation in liver biopsy in patients with chronic HCV infection. Am J Gastroenterol 2002, 97, 2614-2618.

[9] Bedossa P, Dargère D, Paradis V: Sampling variability of liver fibrosis in chronic hepatitis C. Hepatology 2003, 38, 1449-1457.

[10] Bourliere M, Penaranda G, Renou C, Botta-Fridlund D, Tran A, Portal I, Lecomte L, Castellani P, Rosenthal-Allieri M, Gerolami R, Ouzan D, Deydier R, Degott C, Halfon P: Validation and comparison of indexes for fibrosis and cirrhosis prediction in chronic hepatitis $\mathrm{C}$ patients: proposal for a pragmatic approach classification without liver biopsies. J Viral Hepat 2006, 13, 659-670.

[11] Poynard T, Munteanu M, Imbert-Bismut F, Charlotte F, Thabut D, Le Calvez S, Messous D, Thibault V, Benhamou Y, Moussalli J, Ratziu V: Prospective analysis of discordant results between biochemical markers and biopsy in patients with chronic hepatitis C. Clin Chem 2004, 50, 1344-1355.

[12] De Lédinghen V, Vergniol J: Transient elastography for the diagnosis of liver fibrosis. Expert Rev Med Devices 2010, 7, 811-823.

[13] Foucher J, Chanteloup E, Vergniol J, Castéra L, Le Bail B, Adhoute X, Bertet J, Couzigou P, De Lédinghen V: Diagnosis of cirrhosis by transient elastography (FibroScan): a prospective study. Gut 2006, 55, 403-408.

[14] Takeda T, Yasuda T, Nakayama Y, Nakaya M, Kimura M, Yamashita M, Sawada A, Abo K, Takeda S, Sakaguchi H, Shiomi S, Asai H, Seki S: Usefulness of noninvasive transient elastography for assessment of liver fibrosis stage in chronic hepatitis C. World J Gastroenterol 2006, 12, 7768-7773.

[15] Guidelines CP: EASL Clinical Practice Guidelines: management of hepatitis C virus infection. J Hepatol 2011, 55, 245-264.

[16] Ziol M, Handra-Luca A, Kettaneh A, Christidis C, Mal F, Kazemi F, De Lédinghen V, Marcellin P, Dhumeaux D, Trinchet JC, Beaugrand M: Noninvasive assessment of liver fibrosis by measurement of stiffness in patients with chronic hepatitis C. Hepatology 2005, 41, 48-54.

[17] Kettaneh A, Marcellin P, Douvin C, Poupon R, Ziol M, Beaugrand M, De Lédinghen V: Features associated with success rate and performance of FibroScan measurements for the diagnosis of cirrhosis in HCV patients: a prospective study of 935 patients. J Hepatol 2007, 46, 628-634.

[18] Friedrich-Rust M, Ong MF, Herrmann E, Dries V, Rust MF: Real-Time Elastography for Noninvasive Assessment of Liver Fibrosis in Chronic Viral Hepatitis. AJR 2007, 188, 758-764.

[19] Ferraioli G, Tinelli C, Malfitano A, Bello BD, Filice G, Filice C, Above E, Barbarini G, Brunetti E, Calderon W, Di Gregorio M, Lissandrin R, Ludovisi S, Maiocchi L, Michelone G, Mondelli M, Patruno SF, Perretti A, Poma G, Sacchi P, Zaramella M, Zicchetti M: Performance of Real-Time Strain Elastography, Transient Elastography, and aspartate-to-platelet ratio index in the assessment of fibrosis in chronic Hepatitis C. AJR Am J Roentgenol 2012, 199, 19-25.

[20] Yoon JH, Lee JM, Woo HS, Yu MH, Joo I, Lee ES, Sohn JY, Lee KB, Han JK, Choi BI: Staging of hepatic fibrosis: comparison of magnetic resonance elastography and shear wave elastography in the same individuals. KJR 2013, $14,202-212$.

[21] Godfrey EM, Mannelli L, Griffin N, Lomas DJ: Magnetic resonance elastography in the diagnosis of hepatic fibrosis. Seminars in ultrasound CT and MR 2013, 34, 81-88.

[22] Calès P, Oberti F, Michalak S, Hubert-Fouchard I, Rousselet MC, Konaté A, Gallois Y, Ternisien C, Chevailler A, Lunel F: A novel panel of blood markers to assess the degree of liver fibrosis. Hepatology 2005, 42, 1373-1381.

[23] Halfon P, Munteanu M, Poynard T: FibroTest-ActiTest as a non-invasive marker of liver fibrosis. Gastroenter Clin Biol 2008, 32, 22-39.

[24] Guéchot J, Laudat A, Loria A, Serfaty L, Poupon R, Giboudeau J: Diagnostic accuracy of hyaluronan and type III procollagen amino-terminal peptide serum assays as markers of liver fibrosis in chronic viral hepatitis $\mathrm{C}$ evaluated by ROC curve analysis. Clin Chem 1996, 42, 558-563.

[25] Pilette C, Rousselet MC, Bedossa P, Chappard D, Oberti F, Rifflet H, Maiga MY, Gallois Y, Cales P: Histopathological evaluation of liver fibrosis: quantitative image analysis vs. semi-quantitative scores. Comparison with serum markers. J Hepatol 1998, 28, 439-446.

[26] Murawaki Y, Koda M, Okamoto K, Mimura K, Kawasaki H: Diagnostic value of serum type IV collagen test in comparison with platelet count for predicting the fibrotic stage in patients with chronic hepatitis C. J Gastroenterol Hepatol 2001, 16, 777-781.

[27] Murawaki Y, Ikuta Y, Okamoto K, Koda M, Kawasaki H: Serum matrix metalloproteinase-3 (stromelysin-1) concentration in patients with chronic liver disease. J Hepatol 1999, 31, 474-481.

[28] Lichtinghagen R, Michels D, Haberkorn CI, Arndt B, Bahr M, Flemming P, Manns MP, Boeker KH: Matrix metalloproteinase (MMP)-2, MMP-7, and tissue inhibitor of metalloproteinase-1 are closely related to the fibroproliferative process in the liver during chronic hepatitis C. J Hepatol 2001, 34, 239-247. 
[29] Boursier J, Bacq Y, Halfon P, Leroy V, De Ledinghen V, De Muret A, Bourlière M, Sturm N, Foucher J, Oberti F, Rousselet MC, Calès P: Improved diagnostic accuracy of blood tests for severe fibrosis and cirrhosis in chronic hepatitis C. Eur J Gastroenterol Hepat 2009, 21, 28-38.

[30] Naveau S, Gaudé G, Asnacios A, Agostini H, Abella A, Barri-Ova N, Dauvois B, Prévot S, Ngo Y, Munteanu M, Balian A, Njiké-Nakseu M, Perlemuter G, Poynard T: Diagnostic and prognostic values of noninvasive biomarkers of fibrosis in patients with alcoholic liver disease. Hepatology 2009, 49, 97-105.

[31] Castéra L, Vergniol J, Foucher J, Le Bail B, Castera L, Le Bail B: Prospective comparison of transient elastography, Fibrotest, APRI, and liver biopsy for the assessment of fibrosis in chronic hepatitis C. Gastroenterology 2005, 128, 343-350.

[32] Maor Y, Halfon P, Bashari D, Pénaranda G, Morali G, Klar R, Bar-Meir S, Martinowitz U, Oren R: Fibrotest or Fibroscan for evaluation of liver fibrosis in haemophilia patients infected with hepatitis C. Haemophilia 2010, $16,148-154$.

[33] Ferraioli G, Tinelli C, Bello BD, Zicchetti M, Filice G, Filice C, Above E, Barbarini G, Brunetti E, Calderon W, Di Gregorio M, Lissandrin R, Ludovisi S, Maiocchi L, Malfitano A, Michelone G, Mondelli M, Patruno SFA, Perretti AF, Poma G, Sacchi P, Zaramella M: Accuracy of real-time shear wave elastography for assessing liver fibrosis in chronic hepatitis C: A pilot study. Hepatology 2012, 1-9.

[34] Poynard T, Munteanu M, Luckina E, Perazzo H, Ngo Y, Royer L, Fedchuk L, Sattonnet F, Pais R, Lebray P, Rudler M, Thabut D, Ratziu V: Liver fibrosis evaluation using real-time shear wave elastography: applicability and diagnostic performance using methods without a gold standard. J Hepatol 2013, vol. null.

[35] Sebastiani G, Halfon P, Castera L, Pol S, Thomas DL, Mangia A, Di Marco V, Pirisi M, Voiculescu M, Guido M, Bourliere M, Noventa F, Alberti A: SAFE biopsy: A validated method for large-scale staging of liver fibrosis in chronic hepatitis C. Hepatology 2009, 49, 1821-1827.

[36] Lucidarme D, Foucher J, Le Bail B, Vergniol J, Castera L, Duburque C, Forzy G, Filoche B, Couzigou P, De Ledinghen V: Factors of accuracy of transient elastography (fibroscan) for the diagnosis of liver fibrosis in chronic hepatitis C. Hepatology 2009, 49, 1083-1089.

[37] Theodore D, Fried MW, Kleiner DE, Kroner BL, Goedert JJ, Eyster ME, Faust SP, Sherman KE, Kessler CM, Francis C: Aledort LM: Liver biopsy in patients with inherited disorders of coagulation and chronic hepatitis C. Haemophilia 2004, 10, 413-421.

[38] Maor Y, Bashari D, Kenet G, Lubetsky A, Luboshitz J, Schapiro JM, Pénaranda G, Bar-Meir S, Martinowitz U, Halfon P: Non-invasive biomarkers of liver fibrosis in haemophilia patients with hepatitis C: can you avoid liver biopsy? Haemophilia 2006, 12, 372-379.

[39] Posthouwer D, Mauser-Bunschoten EP, Fischer K, Van Erpecum KJ, De Knegt RJ: Significant liver damage in patients with bleeding disorders and chronic hepatitis C: non-invasive assessment of liver fibrosis using transient elastography. JTH 2007, 5, 25-30.

\section{Address for correspondence:}

Marta Kucharska

Department of Infectious Diseases

Hepatology and Acquired Immune Deficiences

Wroclaw Medical University

Koszarowa 5

51-149 Wrocław

Poland

E-mail: mkucharska84@tlen.pl

Conflict of interest: None declared

Received: 1.02.2014

Revised: 4.03.2014

Accepted: 18.06.2014 